\title{
A P4 medicine perspective of gut microbiota and prediabetes: Systems analysis and personalized intervention
}

\begin{abstract}
Qiongrong Huang, ${ }^{1,2}$, Qiaojun Fang, ${ }^{3,4}$ Zhiyuan $\mathrm{Hu}^{1,2,3,4}$
'Fujian Provincial Key Laboratory of Brain Aging and Neurodegenerative Diseases, School of Basic Medical Sciences, Fujian Medical University, Fuzhou 350108, Fujian Province, China; 2Beijing P4 Healthcare Institute, 316 Wanfeng Road, Beijing 100161, China;

${ }^{3}$ CAS Key Laboratory of Standardization and Measurement for Nanotechnology, CAS Key Laboratory for Biomedical Effects of Nanomaterials and Nanosafety, CAS Center for Excellence in Nanoscience, National Center for Nanoscience and Technology of China, Beijing 100190, China;

${ }^{4}$ School of Nanoscience and Technology, Sino-Danish College, University of Chinese Academy of Sciences, Beijing 100049, China
\end{abstract}

\section{ABSTRACT}

Type 2 diabetes (T2D) accounts for approximately $90 \%$ of diabetes worldwide and has become a global public health problem. Generally, individuals go to hospitals and get healthcare only when they have obvious T2D symptoms. While the underlying cause and mechanism of the disease are usually not well understood, treatment is for the symptoms, but not for the disease cause, and patients often continue to progress with more symptoms. Prediabetes is the early stage of diabetes and provides a good time window for intervention and prevention. However, with few symptoms, prediabetes is usually ignored without any treatment. Obviously, it is far from ideal to rely on the traditional medical system for diabetes healthcare. As a result, the medical system must be transformed from a reactive approach to a proactive approach. Root cause analysis and personalized intervention should be conducted for patients with prediabetes. Based on systems medicine, also known as $\mathrm{P} 4$ medicine, with a predictive, preventive, personalized, and participatory approach, new medical system is expected to significantly promote the prevention and treatment of chronic diseases such as prediabetes and diabetes. Many studies have shown that the occurrence and development of diabetes is closely related to gut microbiota. However, the relationship between diabetes and gut microbiota has not been fully elucidated. This review describes the research on the relationship between gut microbiota and diabetes and some exploratory trials on the interventions of prediabetes based on P4 medicine model. Furthermore, we also discussed how these findings might influence the diagnosis, prevention and treatment of diabetes in the future, thereby to improve the wellness of human beings.

Key words: systems medicine, P4 medicine, prediabetes, intervention, microbiome, wellness

\section{INTRODUCTION}

\begin{tabular}{|l|}
\hline Access this article online \\
\hline $\begin{array}{l}\text { Website: } \\
\text { www.intern-med.com }\end{array}$ \\
\hline $\begin{array}{l}\text { DOI: } \\
\text { 10.2478/jim-2020-0020 }\end{array}$ \\
\hline Quick Response Code: \\
\hline \\
\\
\\
\\
\end{tabular}

Prof. Zhiyuan Hu, Fujian Provincia Key Laboratory of Brain Aging and Neurodegenerative Diseases, School of Basic Medical Sciences, Fujian Medical University, Fuzhou 350108, Fujian Province, China.

E-mail: huzy@nanoctr.cn;

Type 2 diabetes (T2D) mellitus is a metabolic disorder that seriously affects human health. ${ }^{[1]}$ Prediabetes, also known as intermediate hyperglycemia, is a state with glycemic index above normal but below diabetes thresholds. It represents a high-risk state which is usually ignored by people. Approximately $5 \%-10 \%$ of people with prediabetes will become diabetic annually, and over $70 \%$ of prediabetic patients will eventually evolve as diabetics in their lifetime. ${ }^{[1,2]}$ According to the World Health
Organization (WHO), prediabetes can be classified into three states: the first state is impaired fasting glucose (IFG) defined as fasting plasma glucose (FPG) of 6.1-6.9 $\mathrm{mmol} / \mathrm{L}$ (with a normal glucose tolerance), the second state is impaired glucose tolerance (IGT) defined as postprandial plasma glucose of $7.8-11.0 \mathrm{mmol} / \mathrm{L}$ based on 2-h oral glucose tolerance test (OGTT) (with a normal FPG), and the third state is a combination of IFG and IGT. ${ }^{[3]}$ Due to the ambiguity of diagnostic criteria and thresholds, asymptomatic prediabetes is likely to be ignored and evolves into T2D. ${ }^{[4]}$ 
However, at the same time, prediabetes provides a valuable opportunity to maintain stability or convert back to health through artificial intervention. A medical concept that is composed of predictive, preventive, personalized, and participatory medical approaches (P4 medicine $)^{[5-12]}$ is expected to reduce the burden of chronic diseases through an in-depth understanding of human interactive systems and underlying mechanisms of chronic disease, and evidence-based personalized interventions. ${ }^{[6]}$ P4 medicine model predicts the trend of health status through monitoring and analysis of omics data, as well as formulates personalized health optimization programs for disease prevention. ${ }^{[13]}$ Through active participation, the P4 medicine model strives to prevent deterioration of health conditions. ${ }^{[6]}$ Based on the P4 medical concept, for prevention or alleviation, prediabetes individuals are usually given personalized recommendations on diet, exercise, stress management, dietary supplements, or physician referral. ${ }^{[13]}$ Among them, modification of diet and exercise belongs to lifestyle intervention. It has been proved that lifestyle modification is the best intervention method for diabetes prevention with evidence of a $40 \%-70 \%$ relative risk reduction, which suggests the indispensability of lifestyle interventions for people with prediabetes. ${ }^{[14]}$ Current guidelines of interventions for diabetes follow the principles of lifestyle improvement, including high dietary fiber diet and frequent excise to achieve moderate weight loss (3\%-7\% weight loss). ${ }^{[15-18]}$ Lifestyle intervention has also proven to be the most appropriate method for Asian prediabetes cohorts. ${ }^{[19,20]}$ Even individuals who have been diagnosed with T2D have the opportunity to achieve a good prognosis and even reverse disease progression by improving their lifestyle. ${ }^{[1,22]}$ There is no doubt that lifestyle change is the most popular, safest, and most effective method of diabetes intervention. ${ }^{[2]}$ Lifestyle intervention supplemented by professional stress management and nutrition guidance will increase the chance of disease improvement.

Systematic analysis of health data can not only tell us whether or not health management is effective, but also provide essential information for performing effective health management. Over the last decade, growing evidence supports that unhealthy gut microbiota (most analyzed from fecal samples) may perturb the host metabolic and immune network and ultimately result in hyperglycemia, insulin resistance, and T2D. ${ }^{[23-25]} \mathrm{It}$ has been proved that gut microbiota varies between prediabetes, diabetes, and healthy people. ${ }^{[26-28]}$ As the disease progresses, the gut microbiota consequently changes. Therefore, it is an interesting question whether the disease condition could be improved by modulating the gut microbiota in prediabetic patients. Several studies based on appropriate diet intervention, such as a high- fiber diet, have successfully improved health status via gut microbiota regulation. ${ }^{[29,30]}$ Other clinical trials with effective interventions through exercise and dietary supplements, like vitamins, prebiotics, probiotics, and synbiotics (combining prebiotics and probiotics), have also observed significant changes in human gut microbiota. ${ }^{[31-34]}$ Thus, it is rational to set the gut microbiota as the pivotal target and the monitoring indicator of health management in prediabetes or diabetes cohort. In this review, based on the concept of P4 medicine, we discuss the feasibility and advantages of targeting the gut microbiota to conduct health management and precise analysis of people with a high risk of diabetes or diagnosed T2D patients. Furthermore, we comment on how to use the P4 medicine model to further quantify diabetes and to build diabetes prevention, monitoring, and treatment system, to improve the health of all.

\section{THE CORRELATION BETWEEN MICROBIOTA AND T2D}

Trillions of microorganisms inhabiting the human gut form a complex interactive community network known as the gut microbiota. The microbiota vastly outnumbers human cells, representing 1-2 kg of body weight. ${ }^{[2]}$ The long-term symbiotic relationship between gut microbiota and mammalian hosts allows microorganisms to provide vital metabolic functions for humans, which the human body cannot perform independently, ${ }^{[35]}$ such as energy homeostasis, building of the gut barrier, signaling in metabolic pathways, formation of the immune system, digestion/absorption of nutrients for the host, ${ }^{[36]}$ and the generation of short-chain fatty acids (SCFAs). ${ }^{[6]}$

Today, many diseases have been identified as related to dysbiosis of the gut microbiota. ${ }^{[27]}$ Particularly, T2D is the first disease suspected of being associated with the microbiome. The study conducted in 345 Chinese individuals firstly proved that the gut microbiota of humans with T2D is different from that of healthy ones. ${ }^{[37]}$ As the pathogenesis of T2D is a continuous process, during which the homeostasis of blood glucose is gradually destroyed, islet $\beta$-cell function is damaged, and insulin resistance is increased, the gut microbiota tends to be disordered. An increasing number of studies reported that prediabetic populations already have significantly different gut microbiota from healthy normal people before they have been diagnosed with diabetes. ${ }^{[1,28,38,39]}$ For a long time, the exploration of the mechanism of diabetes mainly focused on the genome. Although some genetic targets related to the risk of diabetes have been identified, these risk factors generally do not change over time and are unlikely to be affected by artificial intervention. ${ }^{[26]}$ However, the gut 
microbiota, as an important environmental factor that contributes to the progression of diabetes, can be improved by various artificial means.

The P4 medicine model proposes that the chronic disease trajectory and the transition from health to a chronic disease can be divided into four primary stages, including healthy (stage A), the emergence of chronic disease signs (stage B), the emergence of chronic disease symptoms (stage C), and disease diagnosis (stage D) ${ }^{[6]}$ Based on this consensus, the "Predictive" of P4 advocates predicting dysfunction and detecting early disease markers at stage $\mathrm{B}$, and actively intervening before symptoms appear, in order to eliminate the causative factors. ${ }^{[6]}$ "Preventive" advocates the use of systematic medical methods to deeply understand the pathogenesis of chronic diseases, so as to achieve the goal of preventing diseases as early as possible. ${ }^{[6]}$ "Personalized" advocates identifying individuals who are ineffective to treatment based on individual phenotyping and molecular targeting, and further formulating individualized disease treatment plans. ${ }^{[6]}$ "Participatory" means that to make the intervention plan more effective, the individual's attitude toward the intervention plan must undergo a transition from compliance to participation. Through self-tracking, the individuals actively participate in the whole system of personalized treatment and preventive intervention. ${ }^{[6]}$ Generally, after stage B, there are already specific and effective biological markers for evaluation. ${ }^{[6]}$ Omics studies are used to study how the disease develops and progresses; so, the transition period of the disease before stage $\mathrm{C}$ is more valuable. ${ }^{[6]}$ At the same time, interventions and lifestyle management at stage $\mathrm{B}$ or $\mathrm{C}$ are most likely to reverse the disease. ${ }^{[6]}$ Based on the above-mentioned $\mathrm{P} 4$ medicine model, we monitor the gut microbiota and clinical markers to develop the most appropriate personalized intervention plan and encourage participants to participate in the process of predicting and preventing diabetes.

Before the start of health management, participants should conduct a personal lifestyle questionnaire assessment, including diet, exercise, sleep, smoking, drinking, and psychology. At the same time, the coach will ask the participants' background, preferences, expectations, etc. According to the survey report, the coach gave participants tailor-made evidence-based behavior suggestions. ${ }^{[13]}$ Subsequently, the participants will undergo clinical markers and gut microbiota testing for evaluation by the study physician. Through actionable possibilities from their omics data, personalized lifestyle changes and corrections of misunderstandings are ongoing during the initial and entire intervention process. Coaches and physicians will follow-up and evaluate the participants' lifestyles and give corresponding suggestions based on the effects of interventions and adverse reactions.
Some large-scale lifestyle intervention clinical trials use brochures, pedometers, and handheld electronic devices to record detailed lifestyle information. ${ }^{[19,20,40,41]}$ After the intervention, participants perform the same omics test as for the baseline. Such longitudinal omics data research plays an important role in exploring disease mechanisms and testing the improvement of health management on the disease. We analyzed longitudinal gut microbiota and clinical data to identify diabetes-specific gut microbiota interaction networks, intervention-related gut microbiota interaction networks, as well as diabetes risk-related/ protection-related gut microbiota change.

\section{STUDY ON THE ROLE OF GUT MICROBIOTA IN THE PREDIABETIC COHORT BASED ON P4 MEDICINE MODEL}

According to the P4 medicine model and diabetes-related guidelines, the main approaches to the health management of T2D or prediabetes include lifestyle interventions (diet, exercise), dietary supplements (probiotics, prebiotics, synbiotics, vitamins), and drug interventions. Some interventions include high dietary fiber diets, exercise, and metformin, of which the mechanism for improving diabetes had been elucidated. ${ }^{[21,31,37,42]}$

In the process of participating in the studies, participants predicted and prevented the development of T2D through longitudinal omics testing and obtained personal, dense, dynamic data clouds. ${ }^{[13]}$ By personally participating in an evidence-based tailored lifestyle, participants themselves become part of the T2D prediction and prevention system.

\section{LIFESTYLE INTERVENTION}

The National Institute for Health and Care Excellence (NICE) guidelines suggest that individuals with IGT or IFG should firstly receive lifestyle intervention by increasing physical activity, reducing body weight, and health education. ${ }^{[4]}$ Specifically, people with prediabetes should exercise for no less than $150 \mathrm{~min} /$ week and cut down $5 \%-10 \%$ of body weight within a year. ${ }^{[18]}$ Similar suggestions are given by other guidelines from the American Diabetes Association (ADA) that patients should perform a high-intensity exercise at 200-300 min/week and eat a very low-calorie diet (500-750 kcal/day) for 3 months to achieve a weight loss of $>5 \% .{ }^{[15]}$ For patients who succeed in short-term weight loss, long-term $(\geq 1$ year) weight maintenance programs are recommended. ${ }^{[15]}$ Moreover, patients are encouraged to eat a healthy balanced diet, including high-fiber and low-sugar carbohydrates, lowfat dairy products, and oily fish, as well as controlling the 
intake of foods containing saturated and trans-fatty acids. ${ }^{[18]}$ Also, with the reduction of smoking and other unhealthy habits, the benefits from intervention will be enhanced. ${ }^{[18]}$

A 109-person cohort mainly composed of individuals with prediabetes and T2D underwent longitudinal omics profiling for up to 8 years. ${ }^{[22]}$ Integrative personalized tests included deep clinical measures, genome, immunome, transcriptome, proteome, metabolome, microbiome, and wearable monitoring. Participation in research increased participants' awareness of health management. Specific behaviors included active diet and exercise changes without being coached ( $81 \%$ of participants), additional screening, genetic counseling, and personalized medical therapy. Results of self-monitoring health management in this prediabetes cohort showed that $26(23.9 \%)$ progressed, $65(59.6 \%)$ remained stable, and $18(16.5 \%)$ alleviated. Longitudinal data of two prediabetes participants revealed that the development of T2D is related to weight gain and decreased Shannon diversity of the gut microbiota. The proportion of Bacteroidetes in the gut microbiota of both participants increased at the time of the lowest Shannon diversity, causing harm to beneficial bacteria such as the genus Faecalibacterium. Among steady-state plasma glucose (SSPG), a measure of peripheral insulin resistance), FPG, and hemoglobin $\mathrm{A} 1 \mathrm{C}(\mathrm{HbA} 1 \mathrm{c})$, it was found that the diversity of intestinal flora and SSPG showed the strongest correlation. Further, using omics data to predict SSPG, compared to using all omics to achieve an R2 of 0.87 and using only clinical data to achieve an $\mathrm{R} 2$ of 0.59 , using clinical data plus microbiome prediction model can achieve an $\mathrm{R} 2$ of 0.78 . This result shows that the prediction model using only clinical data is unreliable, and the highest accuracy of using all omics predictions has the disadvantages of high cost and complicated operation. The clinical data plus the microbiome can achieve a prediction accuracy of 0.78 , which not only ensures reliability of the prediction, but also saves the cost of testing. A participant in the cohort was diagnosed with B-cell lymphoma during the study. Interestingly, this participant's gut microbiota Shannon diversity changed over time. Shannon diversity began to decline 2 years before diagnosis, reached the lowest at diagnosis, and increased with treatment.

A cross-sectional study of 108 individuals followed for 9 months revealed several health-related findings. ${ }^{[13]}$ Personal data collected included whole genome sequences; clinical tests, metabolome, proteome, and microbiome every 3 months; and frequent activity measurements. Based on the risk factors observed in each participant's clinical markers and genetics, "actionable possibilities" were identified for behavioral coaching to improve their health. The lifestyle recommendations for individuals with prediabetes were based on the Diabetes Prevention Program and personal lifestyle habits. ${ }^{[43]}$ By the last test, significant improvements were observed in vitamin $\mathrm{D}$, mercury, $\mathrm{HbA} 1 \mathrm{c}$, total cholesterol, low-density lipoprotein (LDL) cholesterol, and high-density lipoprotein (HDL) cholesterol. Besides, diabetes risk factors (fasting insulin and homeostatic model assessment of insulin resistance [HOMA-IR]) and inflammation (interleukin 8 [IL-8] and TNF- $\alpha$ ) were significantly improved. Cross-sectional correlation analysis found that microbial a-diversity was negatively correlated with inflammation and immune-related proteins (including [IL-8], FMS-related tyrosine kinase 3 (FLT-3LG), and macrophage colony-stimulating factor 1 [CSF-1]).

\section{DIET}

A high-fiber diet has been well demonstrated to improve the gut microbiota and thus delay or reverse the progression of diabetes. Studies have shown that microbiota plays an essential role to break down indigestible dietary fibers into SCFAs and to synthesize amino acids and vitamins. ${ }^{[2]}$ SCFAs stabilize the glucose level, balance the body energy, and maintain the health of the pancreas. ${ }^{[2]}$ Lack of certain vitamins in the body may also lead to diabetes progression. ${ }^{[34]}$ Dietary fiber can also selectively promote the growth of SCFA-producing strains. ${ }^{[2]}$ When the diversity and abundance of fiber-promoted SCFA-producing strains are further increased, the level of $\mathrm{HbA1c}$ in diabetic patients drops, partly through the increase of glucagonlike peptide-1 (GLP-1) production. ${ }^{[1,44]}$ The dominance of these beneficial strains in the gut also reduces the production of metabolically harmful compounds such as indole and hydrogen sulfide. ${ }^{[21]}$

Previous studies have shown that xylooligosaccharide (XOS) at a dose of $2.8 \mathrm{~g} /$ day can increase Bifidobacterium in healthy individuals. ${ }^{[4]}$ A study that consisted of 16 healthy subjects and 13 prediabetes subjects reported that individuals with prediabetes treated with XOS (2 g/ day) for 8 weeks showed changes in the levels of Blautia, Anaerotruncus, Dialister, and Oscillospira. ${ }^{[4]}$ The abundance of prediabetes-associated Howardella, Slackia, and Enterorhabdus was greatly reduced by XOS in both healthy and prediabetic subjects. ${ }^{[4]}$ The abundance of health-associated Blautia bydrogenotrophica was increased by XOS in both healthy and prediabetes subjects. ${ }^{[4]}$ The reduction of Dialister and Slackia helped to alleviate inflammation, ${ }^{[47,48]}$ and Oscillospira was associated with lower body mass index (BMI). ${ }^{[49]}$ These imply that the XOS diet may be beneficial for alleviating prediabetes symptoms.

Galacto-oligosaccharide (GOS) is another type of dietary fiber. In a prediabetic cohort study consisting of 44 Dutch people, consumption of GOS supplementation $(15 \mathrm{~g} /$ day) for 12 weeks increased the abundance of fecal 
Bifidobacterium, ${ }^{[50]}$ which is consistent with previous studies that used different types of GOS in infants and adults. ${ }^{[1-56]}$ When the substrate is available, Bifidobacterium can produce the end products of lactate and acetate, which belong to SCFAs that can benefit health.

Neither the XOS diet nor the GOS diet intervention that changed gut microbiota showed significant clinical improvement. It suggests the improvement of gut microbiota is earlier than the improvement of clinical markers.

\section{EXERCISE}

A study tracked 217 Mexican individuals consisting of 76 normal people, 54 prediabetics, and 87 diabetics for 1 year. ${ }^{[57]}$ During the period, daily intake of total calories, macronutrients, and sugar was calculated, and physical activity was classified into sedentary, light, moderate, and vigorous levels according to the exercise intensity. ${ }^{[57]}$ Variables that were significantly associated with gut microbiota were found by a Multivariate Association with Linear model (MaAsLin). ${ }^{[57]}$ In terms of dietary intake, lipid intake was negatively related to Kaistobacter. ${ }^{[57]}$ In terms of exercise, physical activity was positively related to Dorea. ${ }^{[57]}$ In terms of weight control, body fat (kg) was positively related to family Enterococcaceae and body weight $(\mathrm{kg})$ was negatively correlated to Fusobacterium genus. ${ }^{[57]}$ From these correlations, we can learn how to design and combine several intervention methods to increase specific beneficial bacteria and reduce harmful ones.

A 12-week high-intensity exercise intervention was performed in a Chinese cohort. ${ }^{[31]}$ This study achieved ideal intervention effects with a modest, but significant weight loss, as well as obvious improvements in insulin sensitivity, lipid profiles, cardiorespiratory fitness, and the levels of adipokines. It was found that changes in gut microbiota were significantly associated with the improvements of HOMA-IR, glucose homeostasis, and insulin sensitivity. Among them, Ruminococcus gnavus, Alistipes shabii, Streptococcus mitis group, Eubacterium ballii, and Escherichia coli showed the strongest associations. Notably, a decrease in Fusobacterium spp. and an increase in Dorea spp. were correlated with multiple markers of insulin resistance observed after the exercise intervention, which is consistent with the result of the above-mentioned Mexican cohort. The Mexican cohort showed that the Fusobacterium spp. were negatively correlated with body weight $(\mathrm{kg})$ and Dorea spp. were positively correlated with exercise intensity.

Surprisingly, the intervention group can be further subdivided into response group $(n=14)$ and non-response group $(n=6)$ according to the degree of HOMA-IR reduction. The abundance and gene expression levels of some strains changed after the exercise intervention. Significant strain-level genomic variation occurred in Bacteroides spp. and Clostridium spp., which were associated with SCFA production. Bifidobacterium spp. and Fusobacteria spp. appeared again in the co-abundance network after the exercise intervention in all exercise participants and the response group, respectively. Dorea spp. were present in all participants of both the exercise and the response groups. The number of positive connections among these butyrate-producing genera within Firmicutes was promoted, the decreased abundance of which is related to obesity and T2D. ${ }^{[42]}$

Pathway enrichment analysis showed that the pathways involved in DNA replication and amino acid metabolism were preferentially enhanced in responders, and that there were differences in glycan biosynthesis and lipid metabolism between the two subgroups. Genes involved in the catabolism of branched-chain amino acids (BCAAs), whose increase promotes insulin resistance, ${ }^{[58]}$ were only significantly increased in responders (Figure 1). Glutamate and sulfur-containing amino acids (SAAs) were excessively increased in non-responders; this change may counteract the effect of exercise on insulin resistance (Figure 1). In the process of carbohydrate and amino acid fermentation, the final reaction products were different in responders and nonresponders due to the different substrate concentrations. Amino acid fermentation formed colonic gases or metabolically detrimental compounds in non-responders and SCFAs in responders (Figure 1). In addition, glutamic acid was converted to gamma aminobutyric acid (GABA; a modulator of glucose homeostasis $\left.{ }^{[59]}\right)$ in responders. However, because its ability to convert to glutamate into GABA was impaired in non-responders, glutamic acid was eventually converted to proline (a harmful end product), causing GABA to decrease in non-responders (Figure 1). Moreover, the glycolysis process showed that genes for synthesizing SCFAs were significantly increased in responders and selectively attenuated in non-responders (Figure 1). In summary, these data shed light on the mechanism of how exercise improves health, and due to the presence of non-responders, personalization of interventions should be emphasized.

Therefore, in order to further realize personalized health management, machine learning is performed using baseline microbial features, achieving a prediction accuracy of 0.747 in an independent validation cohort $(n=30$, perform 12 weeks of high-intensity exercise). The most informative features of gut microorganisms contributing to this classifier include Bacteroides cellulosilyticus, Bacteroides xylanisolvens, and Parabacteroides distasonis. 


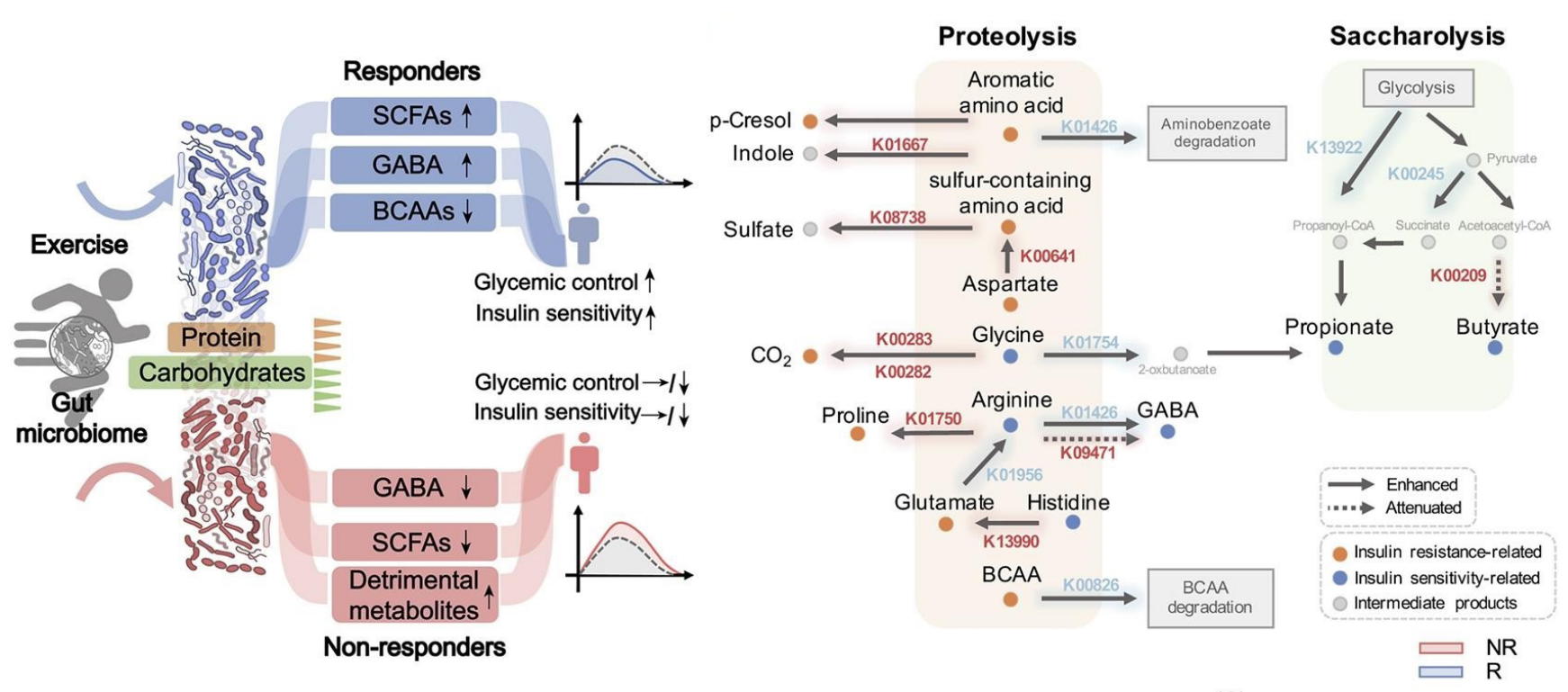

Figure 1: Among responders (R) and non-responders (NR), how high-intensity exercise improves the phenotype through the gut microbiota (left) and their carbohydrate and amino acid fermentation mechanisms, respectively (right). Blue and red indicate selective significant changes in $\mathrm{R}$ and NR, respectively. ${ }^{*} P<0.05,{ }^{* *} P<0.01$, and ${ }^{* * *} P<0.001$ by Wilcoxon rank-sum test between $\mathrm{R}$ and NR. Reprint with permission from Liu et al. ${ }^{[31]}$

\section{INTERVENTION WITH DIETARY SUPPLEMENTS}

For the health management of diabetes, dietary supplements also play an important role. Here we take probiotics, prebiotics, micronutrient, and vitamins as examples.

Compared with healthy people, diabetic patients have an abnormally increased or decreased amount of micronutrients such as vitamins, selenium, copper, magnesium, calcium, folic acid, etc. Individuals with prediabetes also proved to be deficient in a lot of micronutrients. ${ }^{[60-63]}$ The abnormality in the levels of certain essential micronutrients, which is associated with diabetes pathophysiology, reflects the progress of the disease. The long-term imbalance of micronutrients can lead to deterioration and progression of diabetes and increase in the level of oxidative stress. ${ }^{[63-65]}$ Micronutrient-based dietary supplements, including iron and magnesium, have been proven to covariate with microbial composition and functional potential. ${ }^{[66]}$ Taking zinc supplements at $20 \mathrm{mg} /$ day for 12 weeks can significantly improve FPG, OGTT, HOMA-IR, total cholesterol, and LDL levels. ${ }^{[6]}$ When ectopic microbial antigens such as Gram (-) bacteria and lipopolysaccharide (LPS) stimulate host inflammation, coupled with obesity and vitamin deficiency (e.g., vitamin D deficiency [VDD]), it will cause impaired insulin secretion and eventually lead to diabetes. ${ }^{[68]}$ Even so, no practical suggestions on the use of supplements are given for prediabetes or T2D. ${ }^{[69]}$ Additionally, excessive nutrient intake may have adverse effects which lead to increased oxidative stress. ${ }^{[70]}$ Overdoses of vitamin E may even cause intracranial hemorrhage or interact negatively with lipid-lowering drugs. ${ }^{[71]}$ Therefore, it is better to supply essential nutrients through natural foods by eating a balanced diet. However, when certain micronutrients are indeed deficient in a patient, or patients are too weak that their diet is restricted, or uncontrolled progression of diabetes happens, one should consult a dietitian or physician to develop an evidence-based personalized dietary supplements list. ${ }^{[13,71]}$

Ciubotaru et al. reported the gut microbiome changes of two vitamin D-deficient prediabetic groups of African American veterans who underwent vitamin D supplementation with weekly ergocalciferol $(50,000$ IU) over 12 months. ${ }^{[72]}$ One group consisted of people with stable normal glucose tolerance (Gr-1 group) and the other group had stable prediabetes (Gr-2 group). The authors found obvious correlations between the glycemic control and relative abundance of the genera Ruminococcus, Faecalibacterium, and Dialister. The increase in the abundance of the above three bacteria was related to an increase in SCFA production. ${ }^{[73,74]}$ Among them, Dialister spp. also significantly increased after XOS diet intervention. ${ }^{[4]}$ Specific taxa associated with $25(\mathrm{OH})$ $\mathrm{D}$ (the main form of vitamin $\mathrm{D}$ in the human body) from several genera belonging to Lachnospiraceae (e.g., Ruminococcus, Roseburia, Blantia, Dorea) were lower in high vitamin $\mathrm{D}$ quartiles when compared with low vitamin $\mathrm{D}$ quartiles. The results of this study might be biased due to vitamin $\mathrm{D}$ administration. ${ }^{[75]}$ 
In addition, based on current evidence, coenzyme Q10 may assist FPG control, decrease triglycerides, and improve HDL cholesterol in patients with T2D. ${ }^{[76,77]}$ But coenzyme Q10 cannot improve fasting insulin and BMI. ${ }^{176,78,79]}$ The results of different studies on the improvement of $\mathrm{HbA1c}$ are controversial. ${ }^{[7,79]}$ However, most clinical trials of single supplements have failed to elucidate their mechanism of improving diabetes through gut microbiota.

\section{DRUG INTERVENTION}

For diabetics who need medication, personalized medical treatment is also an important part of health management. Drugs are often used in combination with lifestyle intervention. As a first-line drug for diabetes treatment, metformin is the most commonly used drug in clinical trials. In studies that combined lifestyle and metformin interventions, most of the results showed that the contribution of metformin was not as much as lifestyle interventions, i.e., the effects of metformin were not beneficial when combined with lifestyle interventions. ${ }^{[19,80]}$ In a study of pioglitazone intervention, after 18 months of continuous use of pioglitazone at $45 \mathrm{mg}$ / day in a US cohort, the intervention group showed improved liver triglyceride, adipose tissue content, and increased insulin sensitivity of liver and muscle. ${ }^{[81]}$

Metformin is mediated by some gut microbiota for both its therapeutic and adverse reactions. ${ }^{[42]}$ Metformin reverses T2D partly by changing the abundance of Subdoligranulum and Akkermansia (Figure 2). At the same time, microbiota produce more SCFAs, which can improve glucose and energy homeostasis and reduce liver glucose production, appetite, and body weight. The side effects of taking metformin are also inseparable from the change in the microbiota. The depletion of Intestinibacter and the enrichment of Escherichia mean that the ability to resist oxidative stress is reduced, local inflammation is increased, the potential for sulfate metabolism is enhanced, and it can cause gastrointestinal suffering (Escherichia-related LPS synthesis ${ }^{[82]}$ ) and bloating (increased hydrogen sulfide production), which are clinically well-known side effects of metformin treatment (Figure 2). ${ }^{[83]}$ Anti-hypertensives and anti-diabetics were also found to be associated with both microbiome structure and functional capacity. ${ }^{[6]}$

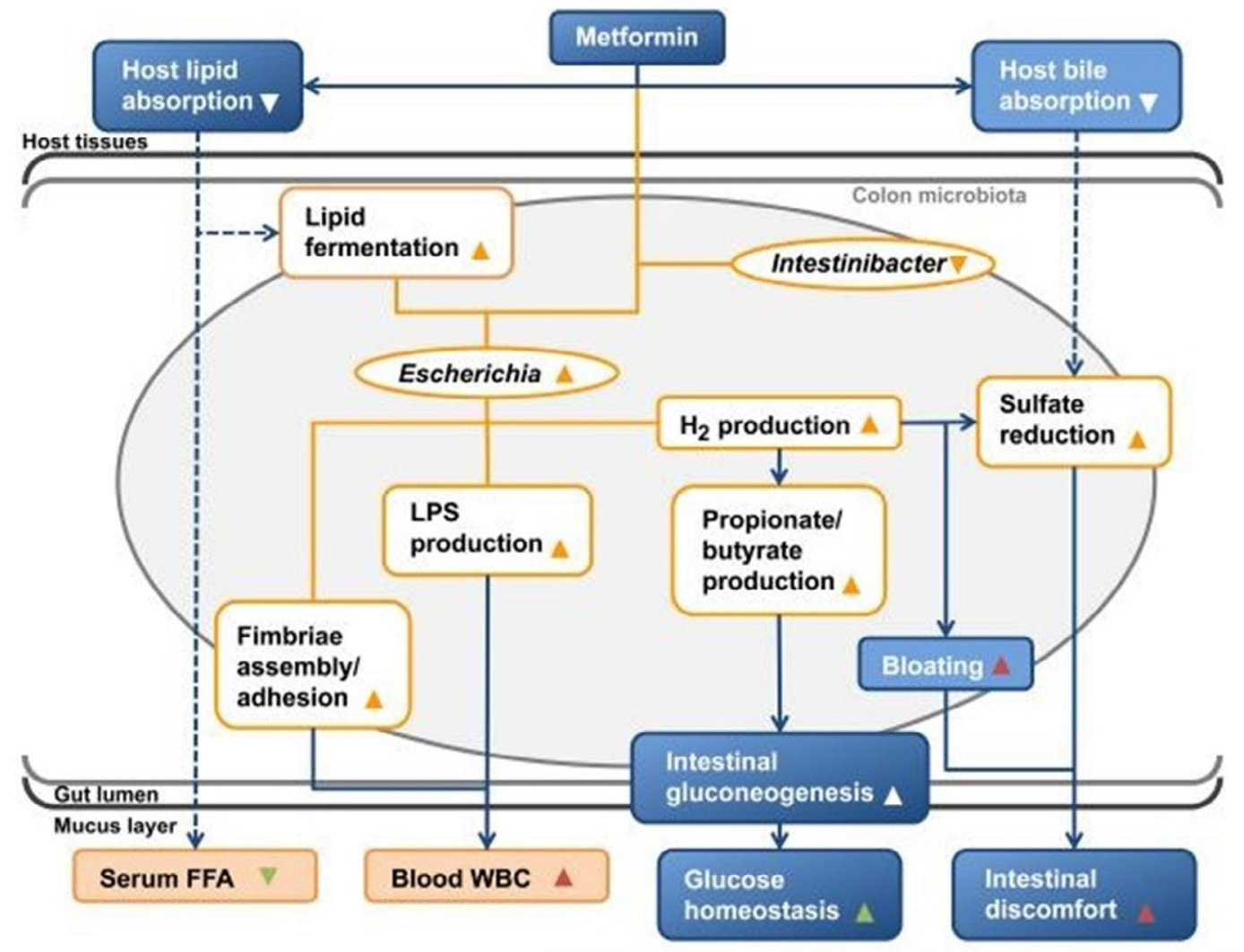

Figure 2: How does metformin produce therapeutic effects (green triangles) and side effects (red triangles) on the host through gut microbiota? The association (orange lines) shown in the illustration between microbial taxa abundance (orange ellipse), microbial functional potential (orange box), blood values (filled orange boxes), and metformin treatment is related to the physiological effects of the host caused by metformin or microorganisms according to literatures (blue boxes and arrows; dashed arrows indicate hypothesized causality). Reprint with permission from Florez et al. ${ }^{[83]}$ 
Huang et al.: A P4 medicine perspective of gut microbiota and prediabetes

\begin{tabular}{|c|c|c|c|c|}
\hline $\begin{array}{l}\text { Intervention } \\
\text { type }\end{array}$ & \multicolumn{4}{|l|}{$\begin{array}{l}\text { (country of } \\
\text { residence) }\end{array}$} \\
\hline \multirow[t]{8}{*}{ Lifestyle } & \multicolumn{2}{|c|}{$\begin{array}{l}109 \text { persons enrichedl }=109 \\
\text { for pre-DM (USA) }\end{array}$} & Self-monitoring lifestyle changes ( 8 years, median 2.8 years) & [22] \\
\hline & 59 pre-DM (USA), & $\mathrm{I}=29, \mathrm{C}=30$ & I, 45 g/day of RS; C, amylopectin (12 weeks) & [84] \\
\hline & $\begin{array}{l}200 \text { pre-DM and } \\
\text { NAFLD (China) }\end{array}$ & $\begin{array}{l}\text { AEx }=50, L C h=50 \\
A E x+L C h=50, C \\
=50\end{array}$ & $\begin{array}{l}\text { AEx, supervised individualized exercise (mainly aerobic } \\
\text { exercise); LCh, individualized diet based on current dietary } \\
\text { intake and body weight; AEx }+ \text { LCh, combined AEx and LCh } \\
\text { program; C, maintaining personal level of physical activity and } \\
\text { habitual eating ( } 6 \text { months) }\end{array}$ & [85] \\
\hline & 641 pre-DM (Japan) & $I=311, C=330$ & $\begin{array}{l}\text { All participants were instructed to reduce total energy intake } \\
\text { and increase physical activity, aiming at a } 5 \% \text { reduction in } \\
\text { body weight with coaching. I, individual instructions } 9 \text { times; } \\
\text { C, individual instructions } 4 \text { times ( } 3 \text { years) }\end{array}$ & {$[20]$} \\
\hline & \multicolumn{2}{|c|}{522 pre-DM (Finland) I $=265, C=257$} & \multicolumn{2}{|l|}{$\begin{array}{l}\text { I, individualized counseling about reducing weight, healthy diet, [41] } \\
\text { and physical activity; C, no specific individualized programs } \\
\text { were offered ( } 6 \text { years) }\end{array}$} \\
\hline & $\begin{array}{l}13 \text { pre-DM, } 16 \\
\text { healthy (USA) }\end{array}$ & $\mathrm{I}=14, \mathrm{C}=15$ & I, 2 g/day XOS; C, 2 g/day placebo (8 weeks) & {$[46]$} \\
\hline & $\begin{array}{l}44 \text { pre-DM (the } \\
\text { Netherlands) }\end{array}$ & $\mathrm{I}=21, \mathrm{C}=23$ & I, $15 \mathrm{~g} /$ day GOS; C, $15 \mathrm{~g} /$ day placebo (12 weeks) & [50] \\
\hline & 39 pre-DM (China) & $I=20, C=19$ & $\begin{array}{l}\text { I, high-intensity supervised exercise; C, sedentary control (12 } \\
\text { weeks) }\end{array}$ & [31] \\
\hline \multirow[t]{6}{*}{ Supplement } & \multicolumn{2}{|c|}{30 pre-DM (Australia)I $=15, C=15$} & $\begin{array}{l}\mathrm{l}, 10 \mathrm{~g} / \text { day prebiotic; } \mathrm{C}, 10 \mathrm{~g} / \text { day placebo }(12 \text { weeks } \\
\text { intervention }+2 \text { weeks washout }+12 \text { weeks crossover } \\
\text { intervention) }\end{array}$ & [86] \\
\hline & \multicolumn{2}{|l|}{$\begin{array}{l}26 \text { pre-DM (New } \\
\text { Zealand) }\end{array}$} & Consumed 2 SunGold kiwifruits/day (12 weeks) & [87] \\
\hline & $\begin{array}{l}200 \text { pre-DM (Sri } \\
\text { Lanka) }\end{array}$ & $\mathrm{I}=100, \mathrm{C}=100$ & $\mathrm{I}$, received zinc at $20 \mathrm{mg} /$ day; $\mathrm{C}$, placebo capsule (12 months) & [19] \\
\hline & 120 pre-DM (Iran) & $\begin{array}{l}\text { Probiotics }=40 \\
\text { Symbiotic }=40 \\
\text { Placebo }=40\end{array}$ & \multicolumn{2}{|l|}{$\begin{array}{l}\text { acidophilus, Bifidobacterium bifidum, Bifidobacterium lactis, and } \\
\text { Bifidobacter longum }\left(1.5 \times 10^{9} \text { for each) with maltodextrin }\right. \\
\text { as the filler; Synbiotics, } 6 \mathrm{~g} / \mathrm{day} \text { comprising the above- } \\
\text { mentioned probiotics plus inulin as a prebiotic; Placebo, } 6 \mathrm{~g} / \text { day } \\
\text { maltodextrin ( } 24 \text { weeks) }\end{array}$} \\
\hline & 200 pre-DM (China) & $I=100, C=100$ & $\begin{array}{l}\text { I, } 840 \mathrm{mg} / \text { day probiotics consisting of live combined } \\
\text { Bifidobacterium longum, L. acidophilus, and Enterococcus } \\
\text { faecalis; C, } 840 \mathrm{mg} / \text { day placebo ( } 24 \text { months) }\end{array}$ & {$[88]$} \\
\hline & $\begin{array}{l}2423 \text { pre-DM (multi- } \\
\text { country) }\end{array}$ & $I=1211, C=1212$ & I, $4000 \mathrm{IU} /$ day of vitamin D; C, placebo (median 2.5 years) & [89] \\
\hline \multirow[t]{4}{*}{$\begin{array}{l}\text { Drug/drug + } \\
\text { lifestyle }\end{array}$} & $\begin{array}{l}93 \text { NGT, } 150 \text { pre- } \\
\text { DM,194 DM (India) }\end{array}$ & $\begin{array}{l}\text { I (LSM, MET, LSM + } \\
\text { MET) }=321, C= \\
116\end{array}$ & $\begin{array}{l}\text { LSM, advice on lifestyle; MET, } 500 \mathrm{~g} / \text { day metformin; LSM }+ \\
\text { MET, LSM plus MET; C, standard healthcare advice (median } 30 \\
\text { months) }\end{array}$ & $0^{[90]}$ \\
\hline & 531 pre-DM (India) & $\begin{array}{l}\mathrm{LSM}=133, \mathrm{MET}= \\
133, \mathrm{LSM}+\mathrm{MET}= \\
129, \mathrm{C}=136\end{array}$ & $\begin{array}{l}\text { LSM, } 30 \mathrm{~min} / \text { day exercise and individualized diet modification; } \\
\text { MET, } 250 \mathrm{mg} \text { metformin twice daily (increase to } 500 \mathrm{mg} \text { twice } \\
\text { daily for some days); LSM + MET, LSM plus MET; C, given } \\
\text { standard healthcare advice (median } 30 \text { months) }\end{array}$ & $e^{[19]}$ \\
\hline & \multicolumn{2}{|c|}{$\begin{array}{l}101 \mathrm{NASH} \text { and either } \mathrm{I}=50, \mathrm{C}=51 \\
\text { pre-DM or DM (USA) }\end{array}$} & I, pioglitazone 45 mg/day; C, placebo (18 months) & [81] \\
\hline & \multicolumn{3}{|c|}{$\begin{aligned} 1945 \text { pre-DM (China) } \mathrm{CLI}=972, \mathrm{ILI}=973 \mathrm{CLI}+\text { placebo, } \mathrm{CLI} \text { with placebo; } \mathrm{CLI}+\text { pioglitazone } \mathrm{HCl} \\
\\
\mathrm{CLI} \text { with pioglitazone } \mathrm{HCl} 30 \mathrm{mg} / \mathrm{day} ; \mathrm{ILI}+\text { placebo, ILI with } \\
\text { placebo; ILI + pioglitazone } \mathrm{HCl} \text {, ILI with pioglitazone } \mathrm{HCl} 30 \\
\text { mg/day ( } 3 \text { years) }\end{aligned}$} & [91] \\
\hline
\end{tabular}




\section{CONCLUSION AND PERSPECTIVE}

For diseases such as diabetes that are clearly controllable through lifestyle changes and whose progression is closely related to one's gut microbiota, it is very appropriate to use the P4 medicine model for early prevention and treatment of individuals. The advancement in gut microbiota detection technology provides a new observation dimension for the exploration of diabetes mechanism and lays a foundation for the discovery of new operable gut microbiota markers. In the diabetic population, Clostridiales sp. SS3/4, Faecalibacterium prausnitziii, Roseburia intestinalis, and Roseburia inulinivorans are depleted, while Bacteroides sp. 20_3, Clostridium hathewayi, Clostridium ramososum, Clostridium symbiosum, and Eggerthella lenta are enriched. ${ }^{[37]}$ Further, identifying the changes in bacterial structure and interaction network caused by the intervention can help discovering the mechanism of how the specific intervention improves host health through microbiota. In addition, it is important to identify which bacterial changes have led to improvements in diabetes and determine the therapeutic components of probiotic. From identifying T2D-related strains to intervention-related strains, and then T2D-relieving related strains, researchers gradually paint a systematic interaction network between the gut microbiota and host.

Intervention for the gut microbiota is still in its infancy. Clinical trials often fail to achieve significant improvements in clinical measurements, ${ }^{[46,50,87]}$ which may be related to the effectiveness of interventions, the length of time required for specific interventions to change gut microbiota, and the strictness of the individual in implementing the new lifestyle in the process of health management. Apart from this, in order to improve the power of using the P4 medicine model to identify the relationship between gut microbiota and diabetes in clinical trials, increasing the sample size and setting up intervention groups ${ }^{[46]}$ and control groups of different quality will be conducive to the credibility and repeatability of the experiment. For dietary interventions, the analysis of nutritional composition may become a breakthrough in establishing the signature of gut microbiota. For exercise interventions, the type and intensity of exercise require further discussion and subdivision. As for oral supplements and drugs, the dosage and frequency need to be emphasized in future experiments. Almost all studies involving the gut microbiota of prediabetic people are focused on bacteria, while eukaryotic microorganisms and viruses are rarely studied.

The ultimate goal of studying the relationship between gut microbiota and diabetes is to build a system where diabetes is accurately classified, monitored, prevented, and treated. Hood et al. and Snyder et al. have found that gut microbiota's a-diversity and microbial structure are related to the underlying inflammation and progression of diabetes. ${ }^{[13,22]}$ However, realization of the ultimate goal must face the challenges arising from technology and increasing people's health awareness. The P4 systems medicine proposes a global, integrated, and quantified approach. ${ }^{[6]}$ The five pillars of P4 medicine are cutting-edge technologies for generating multidimensional data about each person, digital infrastructure for data sharing, personalized data clouds, new analytic techniques and technologies, and systems biology models. ${ }^{[6]}$ The advancement of technology must become a powerful impetus to the progress of chronic disease research. Global comprehensive data cloud sharing breaks the originally limited information and fragmented healthcare and reduces inconsistent or even invalid prescriptions, which greatly promotes multicrossed disciplines, communication between doctors, and improves cost-effectiveness ratio. ${ }^{[13]}$ There are also challenges from sampling, sequencing, bioinformatics analysis, and microbiome functional characterization. ${ }^{[26]}$ Measures such as standardizing the sampling process, ${ }^{[92]}$ establishing minimum information standards, ${ }^{[3]}$ and improving reference species databases are conducive to reducing the error of the results. On the other hand, raising public health awareness requires individuals cared for in the P4 model to start paying attention to their health condition at stage $\mathrm{A}$, and change passive intervention into active prevention, which is also the overarching goal of $\mathrm{P} 4$ health management. ${ }^{[6]}$ Changing the focus from disease to health, including strengthening the health promotion of the patient-free group and developing participatory methods with long-lasting and sustainable impact ${ }^{[6]}$ will be the goal of the joint efforts of health workers across the society.

The presence of exercise non-responders shows that the specific intervention method does not show the same benefit in different individuals. The pattern of gut microbiota shaped by the individual's genetics and the acquired environment is destined to respond to specific interventions. There is a need to establish personalized signatures based on gut microbiota, and guide the corresponding classification, diagnosis, and prognosis plan by the specific microbiota pattern. The multitarget monitoring using omics techniques can guide the intervention with the feedback to modify the intervention to be more accurate and personal. Today, the effect of a certain interventions can be judged with high accuracy based only on the baseline microbiota, ${ }^{[31,37,39]}$ which raises the possibility of maximizing the intervention benefits by improving the gut microbiota. However, it should be pointed out that before using gut microbial signatures, patients must be stratified artificially for treatment to achieve the desired effect of the intervention. ${ }^{[42]}$ This reminds us that a specific gut microbial signature cannot be used in other scenarios, even if this signature has high 
accuracy in that specific scenario. Moreover, the abnormal interaction network between microbiota and human body systems often needs to be regulated from multiple aspects, so the combined use of multiple interventions is a good way. Notably, the presence of many SCFA-producing bacteria, the production of butyric acid, and bifidobacteria have been repeatedly identified as characteristics of the gut microbiota that are beneficial to the health of the host. In the future, researchers will continue to explore more potential gut-based approaches involving microbiota, including the application of personalized dietary supplements (probiotics, prebiotics, or active ingredients in them), FMT, and genetically modified microbiome methods. ${ }^{[25,27]} \mathrm{We}$ expect that technological advances, the popularization of the P4 medicine model, and the specification of clinical trials will speed up the process of human health.

\section{Source of Foundation}

This work was supported by Beijing Municipal Science and Technology Project (Grant No.Z181100001618014); Research Foundation for Advanced Talents of Fujian Medical University (XRCZX2017020, XRCZX2019005); the Strategic Priority Research Program of Chinese Academy of Sciences, Grant No. XDB36000000; National Natural Science Foundation of China (31870992); CASJSPS (Grant No. GJHZ2094); Science and Technology Service Network Initiative of the Chinese Academy of Sciences (Grant No. KFJ-STS-ZDTP-079); and National Natural Science Foundation of China (21775031).

\section{Conflict of Interest}

None declared.

\section{REFERENCE}

1. Zhou WY, Sailani MR, Contrepois K, Zhou YJ, Ahadi S, Leopold SR, et al. Longitudinal multi-omics of host-microbe dynamics in prediabetes. Nature 2019; 569: 663-+.

2. Tabák AG, Herder C, Rathmann W, Brunner EJ, Kivimäki M. Prediabetes: a high-risk state for diabetes development. The Lancet 2012; 379: 2279-90.

3. World Health Organization ID. Definition and diagnosis of diabetes mellitus and intermediate hyperglycaemia: report of a WHO/IDF consultation. . Geneva: World Health Organization 2006.

4. Mangan A, Docherty NG, Le Roux CW, Al-Najim W. Current and emerging pharmacotherapy for prediabetes: are we moving forward? Expert Opin Pharmacother 2018; 19: 1663-73.

5. Vogt H, Hofmann B, Getz L. The new holism: P4 systems medicine and the medicalization of health and life itself. Med Health Care Philos 2016; 19: 307-23.

6. Sagner M, McNeil A, Puska P, Auffray C, Price ND, Hood L, et al. The P4 Health Spectrum - A Predictive, Preventive, Personalized and Participatory Continuum for Promoting Healthspan. Prog Cardiovasc Dis 2017; 59: 506-21.

7. Hood L, Auffray C. Participatory medicine: a driving force for revolutionizing healthcare. Genome Med 2013; 5: 110.
8. Hood L, Balling R, Auffray C. Revolutionizing medicine in the 21st century through systems approaches. Biotechnol J 2012; 7: 992-1001.

9. Hood L, Heath JR, Phelps ME, Lin B. Systems biology and new technologies enable predictive and preventative medicine. Science 2004; 306 640-3.

10. Hood L, Friend SH. Predictive, personalized, preventive, participatory (P4) cancer medicine. Nat Rev Clin Oncol 2011; 8: 184-7.

11. Flores M, Glusman G, Brogaard K, Price ND, Hood L. P4 medicine: how systems medicine will transform the healthcare sector and society. Per Med 2013; 10: 565-76.

12. Hood L. Systems biology and p4 medicine: past, present, and future. Rambam Maimonides Med J 2013; 4: e0012.

13. Price ND, Magis AT, Earls JC, Glusman G, Levy R, Lausted C, et al. A wellness study of 108 individuals using personal, dense, dynamic data clouds. Nature Biotechnology 2017; 35: 747-56.

14. Bansal N. Prediabetes diagnosis and treatment: A review. World J Diabetes 2015; 6: 296-303.

15. 8. Obesity Management for the Treatment of Type 2 Diabetes: Standards of Medical Care in Diabetes-2020.

16. Guess ND. Dietary Interventions for the Prevention of Type 2 Diabetes in High-Risk Groups: Current State of Evidence and Future Research Needs. Nutrients 2018; 10.

17. Stonerock GL, Blumenthal JA. Role of Counseling to Promote Adherence in Healthy Lifestyle Medicine: Strategies to Improve Exercise Adherence and Enhance Physical Activity. Prog Cardiovasc Dis 2017; 59: 455-62.

18. Excellence NIfC. Type 2 diabetes in adults: management. NICE guidelines [NG28] Published December 2015.

19. Ramachandran A, Snehalatha C, Mary S, Mukesh B, Bhaskar AD, Vijay $\mathrm{V}$, et al. The Indian Diabetes Prevention Programme shows that lifestyle modification and metformin prevent type 2 diabetes in Asian Indian subjects with impaired glucose tolerance (IDPP-1). Diabetologia 2006; 49: 289-97.

20. Saito T, Watanabe M, Nishida J, Izumi T, Omura M, Takagi T, et al. Lifestyle modification and prevention of type 2 diabetes in overweight Japanese with impaired fasting glucose levels: a randomized controlled trial. Arch Intern Med 2011; 171: 1352-60.

21. Zhao L, Zhang F, Ding X, Wu G, Lam YY, Wang X, et al. Gut bacteria selectively promoted by dietary fibers alleviate type 2 diabetes. Science 2018; 359: 1151-6.

22. Schussler-Fiorenza Rose SM, Contrepois K, Moneghetti KJ, Zhou W, Mishra T, Mataraso S, et al. A longitudinal big data approach for precision health. Nat Med 2019; 25: 792-804.

23. Aydin O, Nieuwdorp M, Gerdes V. The Gut Microbiome as a Target for the Treatment of Type 2 Diabetes. Curr Diab Rep 2018; 18: 55.

24. Musso G, Gambino R, Cassader M. Interactions between gut microbiota and host metabolism predisposing to obesity and diabetes. Annu Rev Med 2011; 62: 361-80.

25. Brunkwall L, Orho-Melander M. The gut microbiome as a target for prevention and treatment of hyperglycaemia in type 2 diabetes: from current human evidence to future possibilities. Diabetologia 2017; 60: 943-51.

26. Wang J, Jia H. Metagenome-wide association studies: fine-mining the microbiome. Nat Rev Microbiol 2016; 14: 508-22.

27. Vallianou NG, Stratigou T, Tsagarakis S. Microbiome and diabetes: Where are we now? Diabetes Res Clin Pract 2018; 146: 111-8.

28. Zhong H, Ren H, Lu Y, Fang C, Hou G, Yang Z, et al. Distinct gut metagenomics and metaproteomics signatures in prediabetics and treatment-naive type 2 diabetics. EBioMedicine 2019; 47: 373-83.

29. Rivera-Piza A, Lee SJ. Effects of dietary fibers and prebiotics in adiposity regulation via modulation of gut microbiota. Applied Biological Chemistry 2020; 63 .

30. Frazier TH, DiBaise JK, McClain CJ. Gut microbiota, intestinal permeability, obesity-induced inflammation, and liver injury. JPEN J Parenter Enteral Nutr 2011; 35: 14S-20S. 
31. Liu Y, Wang Y, Ni Y, Cheung CKY, Lam KSL, Wang Y, et al. Gut Microbiome Fermentation Determines the Efficacy of Exercise for Diabetes Prevention. Cell Metab 2020; 31: 77-91 e5.

32. Barengolts E. Gut Microbiota, Prebiotics, Probiotics, and Synbiotics in Management of Obesity and Prediabetes: Review of Randomized Controlled Trials. Endocr Pract 2016; 22: 1224-34.

33. Kassaian N, Feizi A, Aminorroaya A, Amini M. Probiotic and synbiotic supplementation could improve metabolic syndrome in prediabetic adults: A randomized controlled trial. Diabetes Metab Syndr 2019; 13: 2991-6.

34. Wilson R, Willis J, Gearry R, Skidmore P, Fleming E, Frampton C, et al. Inadequate Vitamin C Status in Prediabetes and Type 2 Diabetes Mellitus: Associations with Glycaemic Control, Obesity, and Smoking. Nutrients 2017; 9.

35. Neish AS. Microbes in gastrointestinal health and disease. Gastroenterology 2009; 136: 65-80.

36. Galvez J, Rodriguez-Cabezas ME, Zarzuelo A. Effects of dietary fiber on inflammatory bowel disease. Mol Nutr Food Res 2005; 49: 601-8.

37. Qin J, Li Y, Cai Z, Li S, Zhu J, Zhang F, et al. A metagenome-wide association study of gut microbiota in type 2 diabetes. Nature 2012; 490: $55-60$.

38. Allin KH, Tremaroli V, Caesar R, Jensen BAH, Damgaard MTF, Bahl $\mathrm{MI}$, et al. Aberrant intestinal microbiota in individuals with prediabetes. Diabetologia 2018; 61: 810-20.

39. Karlsson FH, Tremaroli V, Nookaew I, Bergstrom G, Behre CJ, Fagerberg $\mathrm{B}$, et al. Gut metagenome in European women with normal, impaired and diabetic glucose control. Nature 2013; 498: 99-103.

40. Harreiter J, Desoye G, van Poppel MNM, Kautzky-Willer A, Dunne F, Corcoy R, et al. The Effects of Lifestyle and/or Vitamin D Supplementation Interventions on Pregnancy Outcomes: What Have We Learned from the DALI Studies? Curr Diab Rep 2019; 19: 162.

41. Tuomilehto J, Lindstrom J, Eriksson JG, Valle TT, Hamalainen H, IlanneParikka $\mathrm{P}$, et al. Prevention of type 2 diabetes mellitus by changes in lifestyle among subjects with impaired glucose tolerance. N Engl J Med 2001; 344: 1343-50.

42. Forslund K, Hildebrand F, Nielsen T, Falony G, Le Chatelier E, Sunagawa $\mathrm{S}$, et al. Disentangling type 2 diabetes and metformin treatment signatures in the human gut microbiota. Nature 2015; 528: 262-6.

43. Diabetes Prevention Program Research G, Knowler WC, Fowler SE, Hamman RF, Christophi CA, Hoffman HJ, et al. 10-year follow-up of diabetes incidence and weight loss in the Diabetes Prevention Program Outcomes Study. Lancet 2009; 374: 1677-86.

44. Willms B, Werner J, Holst JJ, Orskov C, Creutzfeldt W, Nauck MA. Gastric emptying, glucose responses, and insulin secretion after a liquid test meal: effects of exogenous glucagon-like peptide-1 (GLP-1)-(7-36) amide in type 2 (noninsulin-dependent) diabetic patients. J Clin Endocrinol Metab 1996; 81: 327-32.

45. Finegold SM, Li Z, Summanen PH, Downes J, Thames G, Corbett K, et al. Xylooligosaccharide increases bifidobacteria but not lactobacilli in human gut microbiota. Food Funct 2014; 5: 436-45.

46. Yang J, Summanen PH, Henning SM, Hsu M, Lam H, Huang J, et al. Xylooligosaccharide supplementation alters gut bacteria in both healthy and prediabetic adults: a pilot study. Front Physiol 2015; 6: 216.

47. Rocas IN, Siqueira JF, Jr. Characterization of Dialister species in infected root canals. J Endod 2006; 32: 1057-61.

48. Kim KS, Rowlinson MC, Bennion R, Liu C, Talan D, Summanen P, et al. Characterization of Slackia exigua isolated from human wound infections, including abscesses of intestinal origin. J Clin Microbiol 2010; 48: 1070-5.

49. Tims S, Derom C, Jonkers DM, Vlietinck R, Saris WH, Kleerebezem M, et al. Microbiota conservation and BMI signatures in adult monozygotic twins. ISME J 2013; 7: 707-17.

50. Canfora EE, van der Beek CM, Hermes GDA, Goossens GH, Jocken JWE, Holst JJ, et al. Supplementation of Diet With Galacto-oligosaccharides
Increases Bifidobacteria, but Not Insulin Sensitivity, in Obese Prediabetic Individuals. Gastroenterology 2017; 153: 87-97 e3.

51. Depeint F, Tzortzis G, Vulevic J, I'Anson K, Gibson GR. Prebiotic evaluation of a novel galactooligosaccharide mixture produced by the enzymatic activity of Bifidobacterium bifidum NCIMB 41171, in healthy humans: a randomized, double-blind, crossover, placebo-controlled intervention study. Am J Clin Nutr 2008; 87: 785-91.

52. Vulevic J, Juric A, Walton GE, Claus SP, Tzortzis G, Toward RE, et al. Influence of galacto-oligosaccharide mixture (B-GOS) on gut microbiota, immune parameters and metabonomics in elderly persons. Br J Nutr 2015; 114: 586-95.

53. Sierra C, Bernal MJ, Blasco J, Martinez R, Dalmau J, Ortuno I, et al. Prebiotic effect during the first year of life in healthy infants fed formula containing GOS as the only prebiotic: a multicentre, randomised, doubleblind and placebo-controlled trial. Eur J Nutr 2015; 54: 89-99.

54. Vulevic J, Juric A, Tzortzis G, Gibson GR. A mixture of trans-galactooligosaccharides reduces markers of metabolic syndrome and modulates the fecal microbiota and immune function of overweight adults. J Nutr 2013; 143: 324-31.

55. Vulevic J, Drakoularakou A, Yaqoob P, Tzortzis G, Gibson GR. Modulation of the fecal microflora profile and immune function by a novel transgalactooligosaccharide mixture (B-GOS) in healthy elderly volunteers. Am J Clin Nutr 2008; 88: 1438-46.

56. Holscher HD, Faust KL, Czerkies LA, Litov R, Ziegler EE, Lessin H, et al. Effects of prebiotic-containing infant formula on gastrointestinal tolerance and fecal microbiota in a randomized controlled trial. JPEN J Parenter Enteral Nutr 2012; 36: 95S-105S.

57. Chavez-Carbajal A, Pizano-Zarate ML, Hernandez-Quiroz F, Ortiz-Luna GF, Morales-Hernandez RM, De Sales-Millan A, et al. Characterization of the Gut Microbiota of Individuals at Different T2D Stages Reveals a Complex Relationship with the Host. Microorganisms 2020; 8.

58. Newgard CB, An J, Bain JR, Muehlbauer MJ, Stevens RD, Lien LF, et al. A branched-chain amino acid-related metabolic signature that differentiates obese and lean humans and contributes to insulin resistance. Cell Metab 2009; 9: 311-26.

59. Purwana I, Zheng J, Li X, Deurloo M, Son DO, Zhang Z, et al. GABA promotes human beta-cell proliferation and modulates glucose homeostasis. Diabetes 2014; 63: 4197-205.

60. Madhu SV, Raj A, Gupta S, Giri S, Rusia U. Effect of iron deficiency anemia and iron supplementation on HbAlc levels - Implications for diagnosis of prediabetes and diabetes mellitus in Asian Indians. Clin Chim Acta 2017; 468: 225-9.

61. Wang X, Zhang M, Lui G, Chang H, Zhang M, Liu W, et al. Associations of Serum Manganese Levels with Prediabetes and Diabetes among $>/=60$-Year-Old Chinese Adults: A Population-Based Cross-Sectional Analysis. Nutrients 2016; 8.

62. Yadav C, Manjrekar PA, Agarwal A, Ahmad A, Hegde A, Srikantiah RM. Association of Serum Selenium, Zinc and Magnesium Levels with Glycaemic Indices and Insulin Resistance in Pre-diabetes: a Cross-Sectional Study from South India. Biol Trace Elem Res 2017; 175: 65-71.

63. Skalnaya MG, Skalny AV, Tinkov AA. Serum copper, zinc, and iron levels, and markers of carbohydrate metabolism in postmenopausal women with prediabetes and type 2 diabetes mellitus. J Trace Elem Med Biol 2017; 43: 46-51.

64. Salgueiro MJ, Krebs N, Zubillaga MB, Weill R, Postaire E, Lysionek AE, et al. Zinc and diabetes mellitus - Is there a need of zinc supplementation in diabetes mellitus patients? Biological Trace Element Research 2001; 81: 215-28.

65. Jiang R, Manson JE, Meigs JB, Ma J, Rifai N, Hu FB. Body iron stores in relation to risk of type 2 diabetes in apparently healthy women. JAMA 2004; 291: 711-7.

66. Thingholm LB, Ruhlemann MC, Koch M, Fuqua B, Laucke G, Boehm $\mathrm{R}$, et al. Obese Individuals with and without Type 2 Diabetes Show Different Gut Microbial Functional Capacity and Composition. Cell Host 
\& Microbe 2019; 26: 252-+.

67. Ranasinghe P, Wathurapatha WS, Galappatthy P, Katulanda P, Jayawardena R, Constantine GR. Zinc supplementation in prediabetes: A randomized double-blind placebo-controlled clinical trial. J Diabetes 2018; 10: 386-97.

68. Barengolts E. Vitamin D and Prebiotics May Benefit the Intestinal Microbacteria and Improve Glucose Homeostasis in Prediabetes and Type 2 Diabetes. Endocrine Practice 2013; 19: 497-510.

69. Valdes-Ramos R, Guadarrama-Lopez AL, Martinez-Carrillo BE, BenitezArciniega AD. Vitamins and type 2 diabetes mellitus. Endocr Metab Immune Disord Drug Targets 2015; 15: 54-63.

70. Rains JL, Jain SK. Oxidative stress, insulin signaling, and diabetes. Free Radic Biol Med 2011; 50: 567-75.

71. Chertow B. Advances in diabetes for the millennium: vitamins and oxidant stress in diabetes and its complications. MedGenMed 2004; 6: 4.

72. Ciubotaru I, Green SJ, Kukreja S, Barengolts E. Significant differences in fecal microbiota are associated with various stages of glucose tolerance in African American male veterans. Transl Res 2015; 166: 401-11.

73. Khan MT, Duncan SH, Stams AJM, van Dijl JM, Flint HJ, Harmsen HJM. The gut anaerobe Faecalibacterium prausnitzii uses an extracellular electron shuttle to grow at oxic-anoxic interphases. Isme Journal 2012; 6: 1578-85.

74. Walter J, Martinez I, Rose DJ. Holobiont nutrition: considering the role of the gastrointestinal microbiota in the health benefits of whole grains. Gut Microbes 2013; 4: 340-6.

75. Stefanaki C, Peppa M, Mastorakos G, Chrousos GP. Examining the gut bacteriome, virome, and mycobiome in glucose metabolism disorders: Are we on the right track? Metabolism 2017; 73: 52-66.

76. Suksomboon N, Poolsup N, Juanak N. Effects of coenzyme Q10 supplementation on metabolic profile in diabetes: a systematic review and meta-analysis. J Clin Pharm Ther 2015; 40: 413-8.

77. Zhang SY, Yang KL, Zeng LT, Wu XH, Huang HY. Effectiveness of Coenzyme Q10 Supplementation for Type 2 Diabetes Mellitus: A Systematic Review and Meta-Analysis. Int J Endocrinol 2018; 2018: 6484839.

78. Saboori S, Rad EY, Mardani M, Khosroshahi MZ, Nouri Y, Falahi E. Effect of Q10 supplementation on body weight and body mass index: A systematic review and meta-analysis of randomized controlled clinical trials. Diabetes Metab Syndr 2019; 13: 1179-85.

79. Moradi M, Haghighatdoost F, Feizi A, Larijani B, Azadbakht L. Effect of Coenzyme Q10 Supplementation on Diabetes Biomarkers: a Systematic Review and Meta-analysis of Randomized Controlled Clinical Trials. Arch Iran Med 2016; 19: 588-96.

80. Knowler WC, Barrett-Connor E, Fowler SE, Hamman RF, Lachin JM, Walker EA, et al. Reduction in the incidence of type 2 diabetes with lifestyle intervention or metformin. N Engl J Med 2002; 346: 393-403.

81. Cusi K, Orsak B, Bril F, Lomonaco R, Hecht J, Ortiz-Lopez C, et al. Long-Term Pioglitazone Treatment for Patients With Nonalcoholic Steatohepatitis and Prediabetes or Type 2 Diabetes Mellitus: A Randomized Trial. Ann Intern Med 2016; 165: 305-15.

82. Rhee SH. Lipopolysaccharide: basic biochemistry, intracellular signaling, and physiological impacts in the gut. Intest Res 2014; 12: 90-5.

83. Florez H, Pan Q, Ackermann RT, Marrero DG, Barrett-Connor E, Delahanty L, et al. Impact of lifestyle intervention and metformin on healthrelated quality of life: the diabetes prevention program randomized trial. J Gen Intern Med 2012; 27: 1594-601.

84. Marlatt KL, White UA, Beyl RA, Peterson CM, Martin CK, Marco ML, et al. Role of resistant starch on diabetes risk factors in people with prediabetes: Design, conduct, and baseline results of the STARCH trial. Contemp Clin Trials 2018; 65: 99-108.

85. Liu WY, Lu DJ, Du XM, Sun JQ, Ge J, Wang RW, et al. Effect of aerobic exercise and low carbohydrate diet on pre-diabetic non-alcoholic fatty liver disease in postmenopausal women and middle aged men--the role of gut microbiota composition: study protocol for the AELC randomized controlled trial. BMC Public Health 2014; 14: 48.

86. Kellow NJ, Coughlan MT, Savige GS, Reid CM. Effect of dietary prebiotic supplementation on advanced glycation, insulin resistance and inflammatory biomarkers in adults with pre-diabetes: a study protocol for a double-blind placebo-controlled randomised crossover clinical trial. BMC Endocr Disord 2014; 14: 55.

87. Wilson R, Willis J, Gearry RB, Hughes A, Lawley B, Skidmore P, et al. SunGold Kiwifruit Supplementation of Individuals with Prediabetes Alters Gut Microbiota and Improves Vitamin C Status, Anthropometric and Clinical Markers. Nutrients 2018; 10.

88. Yan Q, Li X, Feng B. The efficacy and safety of probiotics intervention in preventing conversion of impaired glucose tolerance to diabetes: study protocol for a randomized, double-blinded, placebo controlled trial of the Probiotics Prevention Diabetes Programme (PPDP). BMC Endocr Disord 2015; 15: 74.

89. Pittas A, Dawson-Hughes B, Staten M. Vitamin D Supplementation and Prevention of Type 2 Diabetes. Reply. N Engl J Med 2019; 381: 1785-6.

90. Snehalatha C, Mary S, Selvam S, Sathish Kumar CK, Shetty SB, Nanditha $\mathrm{A}$, et al. Changes in insulin secretion and insulin sensitivity in relation to the glycemic outcomes in subjects with impaired glucose tolerance in the Indian Diabetes Prevention Programme-1 (IDPP-1). Diabetes Care 2009; 32: 1796-801.

91. Luo Y, Paul SK, Zhou X, Chang C, Chen W, Guo X, et al. Rationale, Design, and Baseline Characteristics of Beijing Prediabetes Reversion Program: A Randomized Controlled Clinical Trial to Evaluate the Efficacy of Lifestyle Intervention and/or Pioglitazone in Reversion to Normal Glucose Tolerance in Prediabetes. J Diabetes Res 2017; 2017: 7602408.

92. Quince C, Walker AW, Simpson JT, Loman NJ, Segata N. Shotgun metagenomics, from sampling to analysis. Nat Biotechnol 2017; 35: 833-44.

93. Yilmaz P, Kottmann R, Field D, Knight R, Cole JR, Amaral-Zettler L, et al. Minimum information about a marker gene sequence (MIMARKS) and minimum information about any $(\mathrm{x})$ sequence $(\mathrm{MIxS})$ specifications. Nat Biotechnol 2011; 29: 415-20.

How to cite this article: Huang Q, Fang Q, Hu Z. A P4 medicine perspective of gut microbiota and prediabetes: Systems analysis and personalized intervention. J Transl Intern Med 2020; 8: 119-30. 